\section{ATRX-mediated chromatin association of histone variant macroH2A1 regulates $\alpha$-globin expression}

\author{
Kajan Ratnakumar, ${ }^{1,2}$ Luis F. Duarte, ${ }^{1,2}$ \\ Gary LeRoy, ${ }^{3,10}$ Dan Hasson, ${ }^{1,4,10}$ \\ Daniel Smeets, ${ }^{5,10}$ Chiara Vardabasso, ${ }^{1,2}$ \\ Clemens Bönisch, ${ }^{6}$ Tianying Zeng, ${ }^{7}$ Bin Xiang, ${ }^{8}$ \\ David Y. Zhang, ${ }^{9}$ Haitao $\mathrm{Li}^{8}{ }^{8}$ Xiaowo Wang, \\ Sandra B. Hake, ${ }^{6}$ Lothar Schermelleh, ${ }^{5,11}$ \\ Benjamin A. Garcia, ${ }^{3}$ and Emily Bernstein ${ }^{1,2,12}$ \\ ${ }^{1}$ Department of Oncological Sciences, ${ }^{2}$ Department of Dermatology, \\ Mount Sinai School of Medicine, New York, New York 10029, USA; \\ ${ }^{3}$ Department of Molecular Biology, Princeton University, Princeton, \\ New Jersey 08544, USA; ${ }^{4}$ Department of Genetics and Genomic \\ Sciences, Mount Sinai School of Medicine, New York, New York \\ 10029, USA; ${ }^{5}$ Department of Biology II, Ludwig Maximilians \\ University Munich, 82152 Martinsried, Germany; ${ }^{6}$ Munich Center \\ for Integrated Protein Science, Adolf-Butenandt Institute, Ludwig- \\ Maximilians University, 80336 Munich, Germany; ${ }^{7}$ MOE Key \\ Laboratory of Bioinformatics, Bioinformatics Division, TNLIST, \\ Department of Automation, Tsinghua University, Beijing 100084, \\ China; ${ }^{8}$ Center for Structural Biology, School of Life Sciences, \\ School of Medicine, Tsinghua University, Beijing 100084, China; \\ ${ }^{9}$ Department of Medicine, Mount Sinai School of Medicine, \\ New York, New York 10029, USA
}

The histone variant macroH2A generally associates with transcriptionally inert chromatin; however, the factors that regulate its chromatin incorporation remain elusive. Here, we identify the SWI/SNF helicase ATRX ( $\alpha$-thalassemia/ $M R, X$-linked) as a novel macroH2A-interacting protein. Unlike its role in assisting $\mathrm{H} 3.3$ chromatin deposition, ATRX acts as a negative regulator of macroH2A's chromatin association. In human erythroleukemic cells deficient for ATRX, macroH2A accumulates at the $\boldsymbol{H B A}$ gene cluster on the subtelomere of chromosome 16, coinciding with the loss of $\alpha$-globin expression. Collectively, our results implicate deregulation of macroH2A's distribution as a contributing factor to the $\alpha$-thalassemia phenotype of ATRX syndrome.

Supplemental material is available for this article.

Received September 20, 2011; revised version accepted January 30, 2012.

The replacement of canonical histones with histone variants contributes to the dynamic nature of chromatin.

[Keywords: macroH2A; histone variant; ATRX; $\alpha$-globin; chromatin remodeling; histone chaperone]

${ }^{10}$ These authors contributed equally to this work.

${ }^{11}$ Present address: Department of Biochemistry, University of Oxford, South Parks Road, Oxford OX1 3QU, United Kingdom.

${ }^{12}$ Corresponding author.

E-mail emily.bernstein@mssm.edu.

Article is online at http://www.genesdev.org/cgi/doi/10.1101/gad.179416.111.
Due to amino acid differences and, in turn, unique posttranslational modifications, histone variants can alter nucleosome structure, stability, and binding of effector proteins. Histone variants have unique genomic localization patterns, and thus specialized roles such as regulating gene expression or chromosome segregation during cell division (Banaszynski et al. 2010). Therefore, the differential genomic incorporation of histone variants directly impacts critical cellular functions.

The histone variant macroH $2 \mathrm{~A}(\mathrm{mH} 2 \mathrm{~A})$ is a vertebratespecific member of the H2A family and is unusual due to the presence of a C-terminal macro domain (Pehrson and Fried 1992). Two different genes encode $\mathrm{mH} 2 \mathrm{~A} 1$ and $\mathrm{mH} 2 \mathrm{~A} 2$ (H2AFY and H2AFY2, respectively), and two splice forms of mH2A1 exist: $\mathrm{mH} 2 \mathrm{~A} 1.1$ and $\mathrm{mH} 2 \mathrm{~A} 1.2$ (Costanzi and Pehrson 2001). mH2A is abundant in heterochromatin, including senescence-associated heterochromatic foci (SAHF) and the inactivated X chromosome (Xi) (Costanzi and Pehrson 1998; Zhang et al. 2005). In vitro studies suggest that the macro domain sterically hinders access of transcription factors to DNA, while mH2A's L1 loop produces inflexible nucleosomes (Angelov et al. 2003; Chakravarthy et al. 2005).

Our group has recently demonstrated a role for $\mathrm{mH} 2 \mathrm{~A}$ isoforms in suppressing melanoma progression, and others have linked $\mathrm{mH} 2 \mathrm{~A}$ expression or its splice patterns to breast and lung cancer (Sporn et al. 2009; Kapoor et al. 2010; Novikov et al. 2011). However, the factors that regulate the association of $\mathrm{mH} 2 \mathrm{~A}$ with chromatin remain obscure. Therefore, identifying regulators of the incorporation of histone variants at distinct genomic loci is key to understanding how chromatin domains are established and maintained and how these may go awry in disease.

A second group of factors contributing to chromatin dynamics are ATP-dependent chromatin remodeling complexes that rearrange or mobilize nucleosomes. Deregulation of members of the SWI/SNF family is implicated in various cancers and mental retardation (MR) syndromes, including ATRX ( $\alpha$-thalassemia/MR, X-linked), (Wilson and Roberts 2011). Mutations in ATRX, predominantly found in the H3K9me3-binding ADD (ATRX-DNMT3DNMT3L) and/or helicase domains, are associated with ATRX syndrome (Higgs et al. 2005; Iwase et al. 2011). This syndrome is characterized by MR and $\alpha$-thalassemia-a loss of $\alpha$-globin gene production (Higgs et al. 2005). However, the mechanisms by which $H B A$ (hemoglobin $\alpha$ ) gene repression occurs are unknown (Higgs et al. 2005).

In addition to its role in regulating gene expression, ATRX acts in concert with Daxx to deposit the H3 variant H3.3 specifically at telomeres (Drane et al. 2010; Goldberg et al. 2010; Lewis et al. 2010), and ATRX deficiency results in loss of telomere integrity (Goldberg et al. 2010; Wong et al. 2010; Heaphy et al. 2011). However, it remains unclear how loss of functional ATRX protein affects the global chromatin landscape of ATRX patients, which may have tissue-specific effects (Berube 2011).

Here, we sought to discover factors involved in regulating mH2A's chromatin association. By isolating mH2A in its chromatin-free state, we identified ATRX as a novel $\mathrm{mH} 2 \mathrm{~A}$ partner. Unlike H3.3, $\mathrm{mH} 2 \mathrm{~A}$ does not interact with Daxx in chromatin-free extracts, suggesting that these two variants interact with unique ATRX complexes. As such, we observed a mutual exclusion between mH2A1.2 and 
H3.3 in the nucleosome. We further demonstrate that ATRX negatively regulates $\mathrm{mH} 2 \mathrm{~A} 1$ chromatin incorporation. Loss of ATRX results in increased $\mathrm{mH} 2 \mathrm{~A} 1$ levels at telomeres, as well as at the $\alpha$-globin locus in erythroleukemic cells, concomitant with reduced transcription of the HBA genes. These data implicate dysregulation of $\mathrm{mH}_{2} \mathrm{~A}^{\prime} \mathrm{s}$ chromatin incorporation as a novel facet of the $\alpha$-thalassemia phenotype of ATRX syndrome.

\section{Results and Discussion}

\section{mH2A interacts with ATRX in a chromatin-free cellular fraction}

To identify factors involved in the regulation of $\mathrm{mH} 2 \mathrm{~A}$ chromatin association, we reasoned they would associate in the soluble nuclear and/or cytoplasmic fractions ("chromatin-free"). Due to their different cellular localization patterns (Fig. 1A; Costanzi and Pehrson 1998) and differential mobility in chromatin, as assayed by fluorescence recovery after photobleaching (FRAP) analysis (Supplemental Fig. 1), we hypothesized that $\mathrm{mH} 2 \mathrm{~A}$ and $\mathrm{H} 2 \mathrm{~A}$ have unique regulatory factors. To this end, we employed a large-scale biochemical strategy to purify chromatin-free GFP-H2A or mH2A1.2-GFP, similar to that used for the identification of the CENPA chaperone HJURP (Fig. 1B; Foltz et al. 2009). Confirmation of immunoprecipitated histones was performed by immunoblotting (Fig. 1C). Following extensive washing and TCA precipitation of the entire immunoprecipitated material, proteins were resolved on a gradient gel, both lanes were excised (10

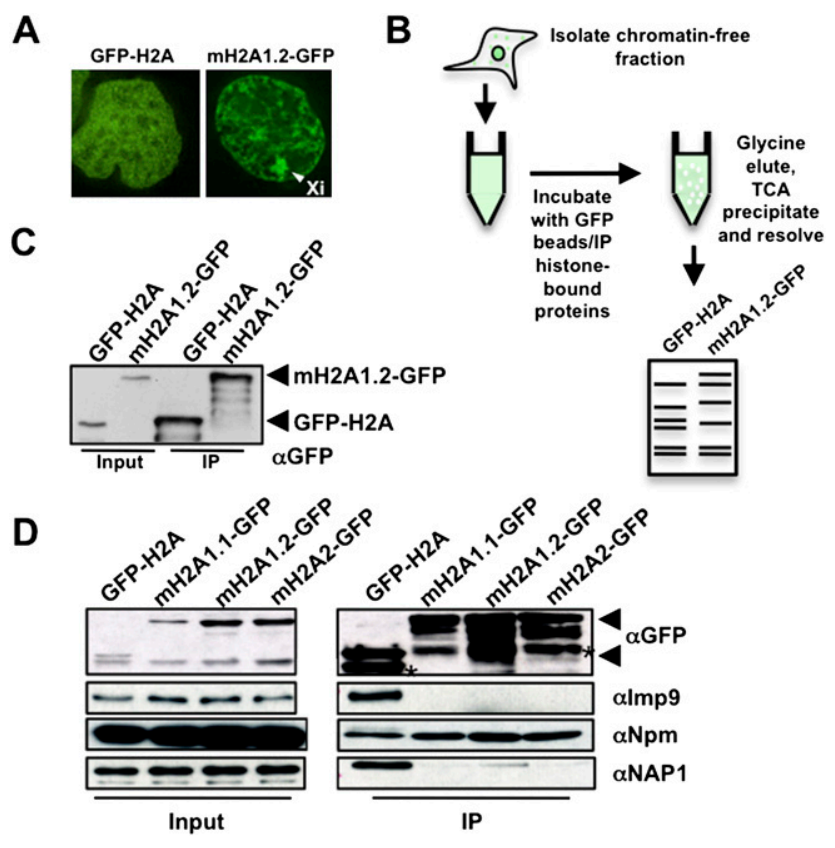

Figure 1. Identification of $\mathrm{mH} 2 \mathrm{~A} 1.2$ chromatin-free interacting factors. (A) Fluorescence microscopy of HEK293 cells stably expressing GFP-H2A and mH2A1.2-GFP. Arrowhead indicates Xi. (B) Procedure used to isolate chromatin-free $\mathrm{H} 2 \mathrm{~A}$ - and mH2A1.2-interacting factors. $(C) \alpha \mathrm{GFP}$ immunoblot confirms expression and immunoprecipitation of histones in stable cell lines. (D) Immunoblots of Imp9, Npm, and NAP1 association with GFP-H2A or mH2A-GFP isoforms. Arrows on all $\alpha$ GFP blots indicate GFP-H2A (bottom) and $\mathrm{mH} 2 \mathrm{~A}-$ GFP (top), and asterisks indicate degradation products. slices per lane), and mass spectrometry (MS) analysis on all gel slices was performed (Fig. 1B; Supplemental Fig. 2A).

From the proteins retrieved (Supplemental Table 1), we focused on factors that regulate chromatin association of histones, including nuclear import factors (Supplemental Fig. 2B). For example, kap114p mediates the nuclear import of $\mathrm{H} 2 \mathrm{~A} / \mathrm{H} 2 \mathrm{~B}$ in Saccharomyces cerevisiae (Mosammaparast et al. 2005), and accordingly, we identified the mammalian homolog Importin 9 (Imp9) as an H2A import factor. We validated Imp9 by immunoblots and independent MS experiments where specific bands were excised (Fig. 1D; Supplemental Fig. 2C,D). These data suggest that the mechanism of histone import is evolutionarily conserved and, importantly, validate our technical approach.

As we were interested in factors that directly regulate chromatin association of $\mathrm{mH} 2 \mathrm{~A} 1$, we further focused on histone chaperones (De Koning et al. 2007; Park and Luger 2008). We identified peptides from NAP1, SET/ TAF-I, nucleolin (Ncl), and nucleophosmin (Npm) in our MS analysis (Supplemental Figs. 2A, 3A). Some of these factors, such as Ncl and Npm, were present in both the GFP$\mathrm{H} 2 \mathrm{~A}$ and mH2A1.2-GFP immunoprecipitations, and we reasoned they were general histone-interacting proteins. Indeed, this is the case (Dunleavy et al. 2009; Gaume et al. 2011), as confirmed by our immunoblots (Fig. 1D). We further confirmed specificity of NAP1 for H2A by immunoblot (Fig. 1D), as reported (Park and Luger 2008). Due to the lack of specificity for $\mathrm{mH} 2 \mathrm{~A}$, these factors were unlikely candidates for regulating its chromatin association.

Of the potential histone chaperones identified by MS, the SWI/SNF chromatin remodeling protein ATRX interacted uniquely with mH2A1.2 (Supplemental Fig. 3A). Nineteen peptides spanning the entire ATRX protein were identified (Supplemental Fig. 3B). We confirmed this interaction using the immunoprecipitation protocol as performed for MS analysis (Fig. 2A) and via an alternative chromatin-free approach with similar results (Supplemental Fig. 3C; Mendez and Stillman 2000). Of note, we did not detect $\mathrm{H} 3$ in the immunoprecipitation from either protocol, suggesting that the tagged histones in our extracts were indeed chromatin-free (nonnucleosomal) and that the $\mathrm{mH} 2 \mathrm{~A}-\mathrm{ATRX}$ interaction is independent of $\mathrm{H} 3$ binding (Fig. 2A). H2B peptides were detected via MS, suggesting the presence of $(\mathrm{m}) \mathrm{H} 2 \mathrm{~A}-\mathrm{H} 2 \mathrm{~B}$ dimers.

We next examined the interaction between ATRX and $\mathrm{mH} 2 \mathrm{~A}$ isoforms in chromatin-free extracts. ATRX interacts with all $\mathrm{mH} 2 \mathrm{~A}$ isoforms (Fig. $2 \mathrm{~B}$ ), suggesting the interaction occurs through the highly conserved H2A domain. As expected, Imp9 preferentially associated with $\mathrm{H} 2 \mathrm{~A}$, and Parp1 uniquely interacted with $\mathrm{mH} 2 \mathrm{~A} 1.1$, as previously described (Timinszky et al. 2009). We then performed reverse coimmunoprecipitation (co-IP) experiments in HEK293 cells transfected with GFP-tagged ATRX fragments (Supplemental Fig. 4). Using whole-cell extracts, we narrowed down the region required for $\mathrm{mH} 2 \mathrm{~A} 1$ binding to amino acids $1-841$ of ATRX (Fig. 2C). As positive controls, this fragment also bound $\mathrm{H} 3$, while a construct spanning the middle region of ATRX (800-1670) bound Daxx (Tang et al. 2004). We next validated this interaction in chromatin-free immunoprecipitations (Fig. 2D). Interestingly, the $\mathrm{N}$ terminus of ATRX contains an ADD domain, an HP1-binding "PxVxL" motif, an $\alpha$-helical region, and an acid-rich motif (B Xiang and $\mathrm{H} \mathrm{Li}$, unpubl.). It will be key to decipher whether mH2A-ATRX binding is direct and, if so, which domains mediate this interaction. 
A

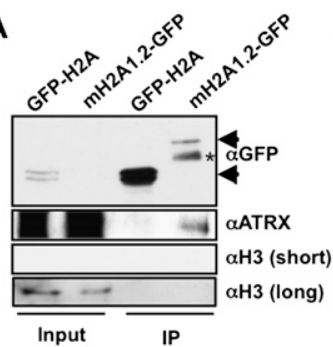

B

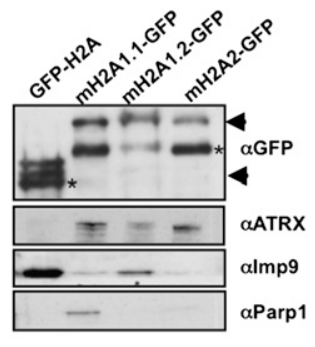

C

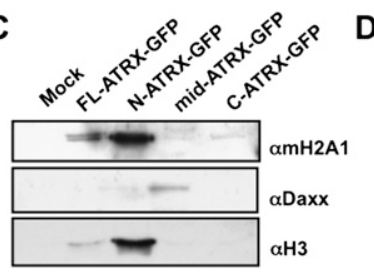

D

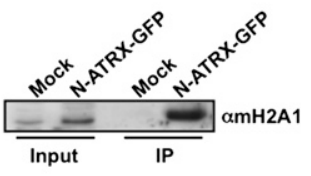

Figure 2. ATRX interacts with $\mathrm{mH} 2 \mathrm{~A}$ isoforms in chromatin-free extracts. $(A, B)$ Immunoblots of GFP and ATRX from chromatin-free extracts. The absence of $\mathrm{H} 3$ in immunoprecipitations confirms chromatin-free interactions; see the long exposure. (B) Co-IP of GFP-H2A and all mH2A isoforms in chromatin-free extracts for ATRX. Immunoblots for Imp9 and Parp1 demonstrate chromatin-free interactions. (C) Whole-cell immunoprecipitations of GFP-tagged ATRX constructs (full-length, N-terminal [1-841], middle region [800-1670], and C-terminal [1670-2492]), followed by mH2A1, H3, and Daxx immunoblots. (D) Chromatin-free co-IP of N-ATRX-GFP with mH2A1.

\section{$\mathrm{mH} 2 \mathrm{~A}$ and $\mathrm{H3} .3$ are in distinct ATRX complexes}

While ATRX deposits $\mathrm{H} 3.3$ at telomeres via its interaction with Daxx (Drane et al. 2010; Goldberg et al. 2010; Lewis et al. 2010), we were unable to detect an interaction between mH2A isoforms and Daxx via immunoblot (Fig. 3A), and Daxx peptides were not detected in our MS analysis (Supplemental Table 1). However, Daxx indeed interacts specifically with H3.3 in chromatin-free extracts (Fig. 3B). These results suggest that $\mathrm{H} 3.3$ and $\mathrm{mH} 2 \mathrm{~A}$ are in distinct ATRX complexes, distinguished by the presence of Daxx.

Based on the above, we hypothesized that $\mathrm{mH} 2 \mathrm{~A} 1$ and H3.3 exist in mutually exclusive nucleosomes. To test this directly, we immunoprecipitated mononucleosomes from H2A- and mH2A1.2-Flag-tagged HeLa cells (Fig. 3C) and performed MS analysis to determine their $\mathrm{H} 3$ variant composition. While H2A-containing nucleosomes contain $>40 \% \mathrm{H} 3.3$, those of $\mathrm{mH} 2 \mathrm{~A} 1.2$ contain $\sim 4 \%$ (Fig. 3D). This suggests that distinct factors or complexes regulate chromatin association of $\mathrm{mH} 2 \mathrm{Al}$ and $\mathrm{H} 3.3$, and we hypothesized that while ATRX-Daxx deposits H3.3 into chromatin, ATRX inhibits mH2A chromatin incorporation.

Loss of ATRX results in altered levels of $\mathrm{mH} 2 \mathrm{~A} 1$ in chromatin

To test this hypothesis, we probed the effects of ATRX depletion on $\mathrm{mH} 2 \mathrm{~A}$ chromatin association. We engineered HEK293 cells to stably express shRNAs targeting ATRX or luciferase (control). We selected two shRNA lines that induced significant knockdown (sh90 and sh92) (Fig. 4A; Supplemental Fig. 5A) for further experiments and ensured that mH2A levels were unaffected (Fig. 4A). This knockdown may mimic ATRX syndrome, as patients with ATRX mutations have reduced protein levels or decreased enzymatic activity of the helicase domain (Berube 2011; Mitson et al. 2011).

We next inquired whether loss of ATRX altered mH2A chromatin association. Loss of ATRX resulted in a global increase of $\mathrm{mH} 2 \mathrm{Al}$ in chromatin, while total cellular levels remained constant (Fig. 4B), implicating ATRX as a negative regulator of $\mathrm{mH} 2 \mathrm{~A}^{\prime} \mathrm{s}$ chromatin incorporation. Quantitative MS (qMS) analysis on histones extracted from chromatin (Plazas-Mayorca et al. 2009) of shluc and sh92 HEK293 cells revealed that loss of ATRX results in $\sim 30 \%$ more mH2A1 in chromatin (Fig. 4C; Supplemental Fig. 6). To examine the dynamics of mH2A1.2-GFP in the absence of ATRX in vivo, we performed FRAP using stable
shRNA lines generated in HeLa1.2.11 cells (Supplemental Fig. 5B,C). FRAP studies revealed a decrease in fluorescence recovery of mH2A1.2 in sh92 cells, suggesting a more stable association of mH2A1.2 with chromatin upon loss of ATRX (Fig. 4D).

\section{ATR $X$ regulates $m H 2 A 1$ incorporation at telomeres and the $\alpha$-globin cluster}

As mH2Al chromatin association increased in ATRXdepleted cells, we inquired which genomic regions are enriched in mH2A1. Because ATRX localizes to telomeres (Goldberg et al. 2010; Wong et al. 2010), we hypothesized that global increase of mH2A1 might, in part, be a result of telomeric accumulation. Chromatin immunoprecipitation (ChIP) followed by Southern blot demonstrated increased association of $\mathrm{mH} 2 \mathrm{Al}$ with telomeres in ATRX knockdown lines of HEK293 and erythroleukemic K562 cells, which express $\alpha$-globin (Fig 5B; Supplemental Fig. 7; see below). The weaker of the two ATRX knockdowns

A
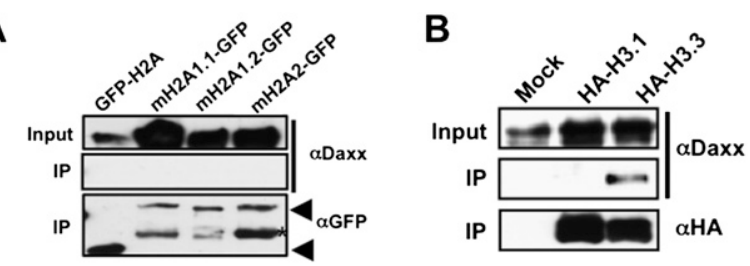

C
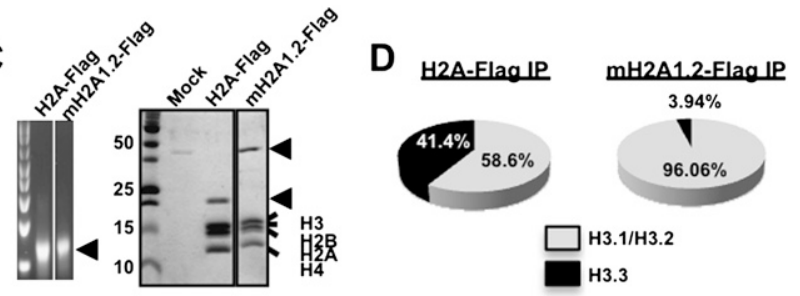

Figure 3. $\mathrm{mH} 2 \mathrm{~A}$ and $\mathrm{H} 3.3$ are in distinct ATRX complexes. Co-IPs of chromatin-free association of $\mathrm{H} 2 \mathrm{~A}$ and $\mathrm{mH} 2 \mathrm{~A}$ isoforms $(A)$ or $\mathrm{H} 3$ variants $(B)$ with Daxx. Immunoblots detected the presence of Daxx specifically with H3.3. (C) Ethidium bromide-stained (left) and Coomassie-stained (right) mononucleosomes from H2A-Flag and mH2A1.2-Flag immunoprecipitations. (D) $\mathrm{H} 3$ composition of $\mathrm{H} 2 \mathrm{~A}-$ Flag and mH2A1.2-Flag mononucleosomes as analyzed by MS. The pie chart depicts the abundance of H3.1/H3.2 (gray) and H3.3 (black) in immunoprecipitated nucleosomes. 
A

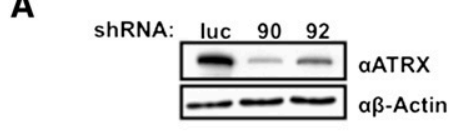

B

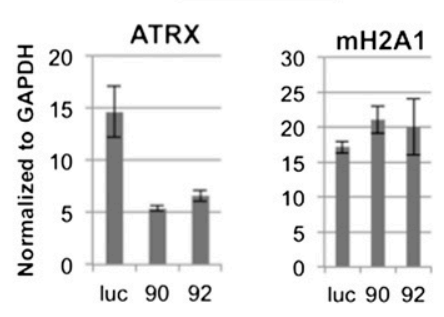

C

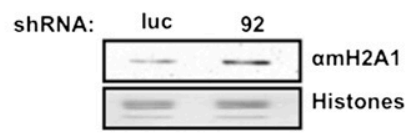

D

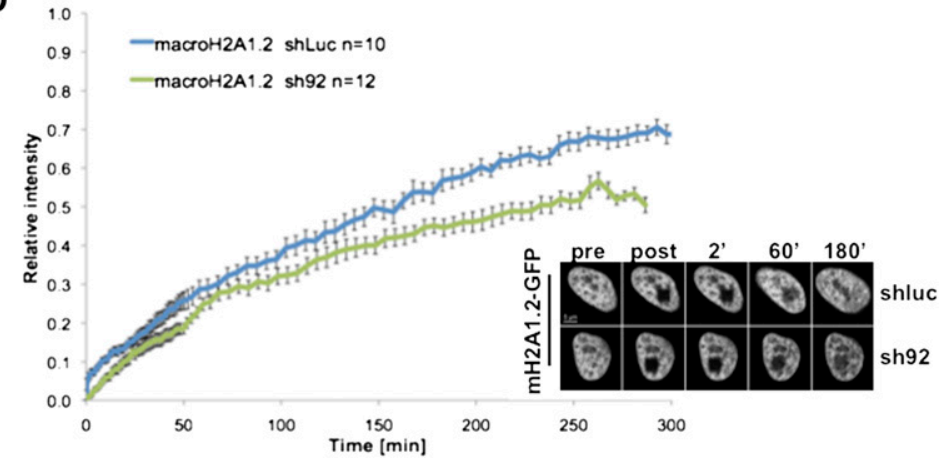

Figure 4. ATRX knockdown results in increased levels and stability of mH2A1 in chromatin. (A) shRNA-mediated knockdown of ATRX (sh90 and sh92) in HEK293 cells results in the loss of ATRX protein and mRNA, compared with shluc, without affecting mH2A1 mRNA. $\beta$-Actin was used for loading. $(B)$ Loss of ATRX results in accumulation of $\mathrm{mH} 2 \mathrm{~A} 1$ in chromatin (top panel), and the whole-cell content remains unaffected (bottom panel). H2B was used for loading. (C) mH2A1 immunoblot of chromatin-extracted histones analyzed by qMS. ( $D$ left) Quantitation of FRAP experiments indicates slower recovery of mH2A1.2GFP in HeLa1.2.11 cells depleted of ATRX (sh92; green line) compared with control (shluc; blue line). (Right) Representative images of FRAP time series preand post-bleach are shown.

(sh92) revealed $>10$-fold $\mathrm{mH} 2 \mathrm{~A} 1$ enrichment in $\mathrm{K} 562$ cells (Fig. 5B), suggesting partial loss of function may have significant consequences, possibly akin to the syndrome (Berube 2011; Mitson et al. 2011). While currently unclear, ATRX-mediated regulation of $\mathrm{mH} 2 \mathrm{~A}$ levels at telomeres may help to ensure telomeric integrity.

Next, we queried whether loss of ATRX affects expression of the subtelomeric $\alpha$-globin gene cluster on human chromosome 16 in erythroid cells. Upon ATRX knockdown in K562 cells (Fig. 5A; Supplemental Fig. 8A), HBA mRNA and protein levels were dramatically reduced (Fig. 5C). Other genes in this region, including the ATRX target NME4 (Law et al. 2010), were also transcriptionally decreased, while $C D K 8$ on chromosome 13 was unaffected (Supplemental Fig. 8B; Kapoor et al. 2010).

As mH2A is generally transcriptionally repressive, we hypothesized that deregulation of $\mathrm{mH} 2 \mathrm{~A}$ nucleosome occupancy represses the $H B A$ genes, which are silenced in ATRX patients by undefined mechanisms (Higgs et al. 2005; Berube 2011). To examine mH2A1 distribution across the $\alpha$-globin cluster, we performed native ChIP-seq in shluc and sh92 K562 cells (Supplemental Fig. 9A). We obtained $56,540,184$ reads for shluc, $67,219,237$ for sh92, and $148,165,330$ for input DNA using Illumina Hi-Seq (Supplemental Fig. 9B). Analyses were performed on normalized alignments (to the total number of alignments) to account for the different number of reads between samples. We found $\mathrm{mH} 2 \mathrm{~A} 1$ to be generally (1) excluded from transcriptional start sites (TSSs) and (2) in broad domains both upstream of and downstream from the TSS, particularly at genes transcribed at low levels. This supports its role as a repressive variant and is consistent with ChIPchip studies (Supplemental Fig. 10A; Buschbeck et al. 2009; Gamble et al. 2010).

While K562 cells express $\alpha$-globin, levels are lower than primary erythroblasts (D Higgs, pers. comm.). In accordance, we observed a distinct mH2A1 domain at the $\alpha$-globin cluster in K562 cells, however, with more significant peaks of enrichment in sh92 cells (Fig. 5D; Supplemental Fig. 11A). These data strongly suggest that mH2A1 is enriched at this gene cluster in the absence of ATRX. Globally, the total number of base pairs covered by mH2A1 significant peaks is $20 \%$ higher in sh92 cells than shluc cells. This is likely due to mH2A1 redistribution, as only $\sim 45 \%$ of the peaks are shared between shluc and sh92 (Supplemental Fig. 9B,C). Interestingly, we observed a global anticorrelation of mH2A1 domains and ATRX peaks, which are generally concentrated around TSSs (Fig. 5D; Supplemental Fig 10B,C; Law et al. 2010), suggesting that ATRX prevents mH2Al chromatin incorporation. Finally, by quantitative PCR (qPCR) analysis of native ChIP DNA and crosslinked ChIP DNA, we observed marked increase at regions in the $\alpha$-globin cluster previously reported to be mH2A1-enriched (Supplemental Fig. 11B,C; Gamble et al. 2010). Taken together, these data suggest that mH2A1 is specifically deposited at the $\alpha$-globin gene cluster in an ATRX-mediated fashion.

Here, we took an unbiased approach to identify factors that specifically associate with $\mathrm{mH} 2 \mathrm{~A}$ in its chromatin-free state. We identify ATRX as a negative regulator of $\mathrm{mH} 2 \mathrm{~A} 1$ chromatin incorporation, particularly at telomeres and the $\alpha$-globin locus. Such regulation of histone incorporation via inhibitory factors remains relatively unexplored. A recent study identified INO80, also an ATP-dependent chromatin remodeling enzyme, as a negative regulator of H2A.Z nucleosomal incorporation (Papamichos-Chronakis et al. 2011). In the absence of INO80, genomic distribution of H2A.Z is perturbed, resulting in a reduced response to transcriptional changes. Whether ATRX directly interacts with $\mathrm{mH} 2 \mathrm{~A}$ to evict this histone variant from chromatin or inhibit its deposition remains unclear. If the interaction is direct, dissecting the surfaces that mediate binding will be important. In addition, it remains formally possible that alternatively spliced or modified forms of ATRX differentially interact with mH2A and H3.3 (Berube et al. 2000; Garrick et al. 2004). We look forward to future studies addressing the positive and negative regulation of histone variants within the chromatin template, the factors involved, and the underlying mechanisms.

Finally, our data point toward a novel mechanism by which the histone variant $\mathrm{mH} 2 \mathrm{Al}$ is involved in the $\alpha$-thalassemia phenotype of ATRX patients. While the ATRX-Daxx complex has been shown to deposit H3.3, the genomic localization and function of $\mathrm{H} 3.3$ have yet to be explored in the context of ATRX syndrome. Here, we 
A

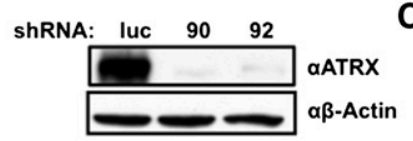

shRNA

D
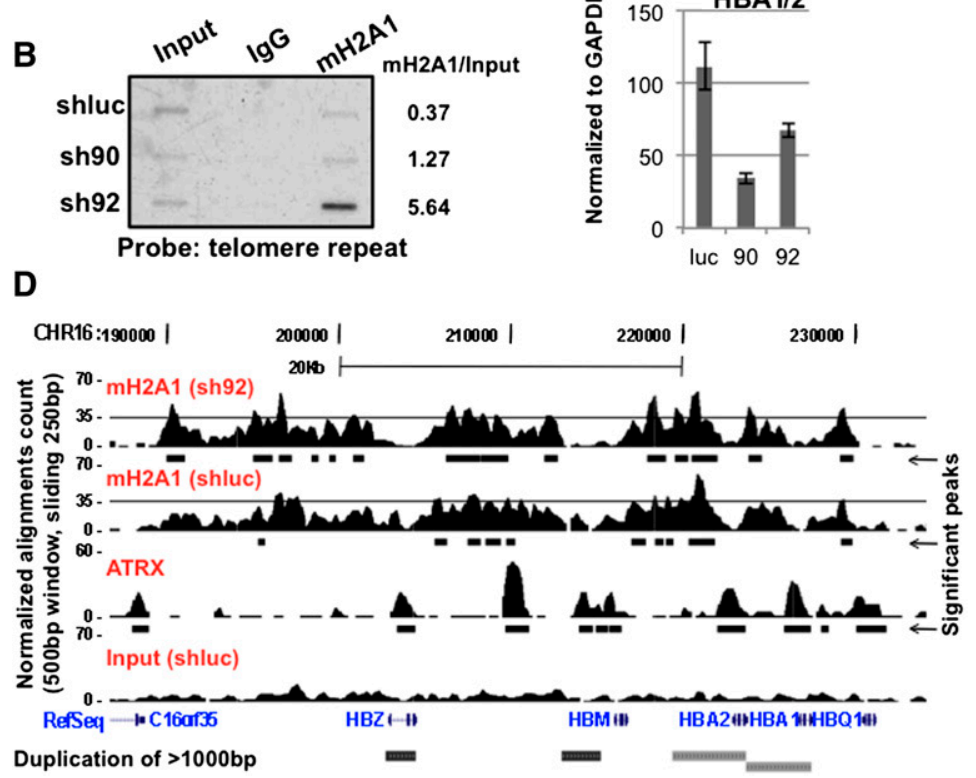

Figure 5. ATRX loss results in increased association of $\mathrm{mH} 2 \mathrm{Al}$ at telomeres and the $\alpha$-globin cluster, concomitant with loss of $\alpha$-globin expression. $(A)$ shRNAmediated knockdown of ATRX (sh90 and sh92) in K562 cells. $\beta$-Actin was used for loading. $(B) \mathrm{ChIP}$ reveals increased association of $\mathrm{mH} 2 \mathrm{Al}$ with telomeres in the absence of ATRX. (Right) Densitometry quantitation. One of two biological replicates is shown. $(C)$ Loss of ATRX results in decreased $\alpha$-globin protein and HBA mRNA. $(D)$ Capture of the University of California at Santa Cruz Genome Browser showing an $\sim 50-\mathrm{kb}$ region around the $\alpha$-globin locus. ChIP-seq-enriched peaks are shown for mH2A1 (sh92 and shluc), ATRX (Law et al. 2010), and input (shluc). Significant peaks (MACS) are shown below each panel as black bars. (Bottom) RefSeq annotated genes. The threshold line was set at 35 to facilitate visualization. beads were washed and boiled in Laemmli loading buffer, and proteins were resolved by PAGE.

\section{Chromatin fractionation, histone acid extraction, and immunoblots}

Chromatin fractionation was performed as described (Mendez and Stillman 2000). Histone acid extraction was performed as described (Kapoor et al. 2010), with the exception that isolated chromatin was treated with $\mathrm{H}_{2} \mathrm{SO}_{4}$. Immunoblots were performed with the following antibodies: $\alpha$ GFP (Roche, 11814 460 001), $\alpha$ ATRX (Santa Cruz Biotechnology, sc15408), $\alpha$ IMP9 (Abcam, ab52605), $\alpha$ Nap1L1 (Abcam, ab33076), $\alpha$ Npm (Chemicon, MAB4500), $\alpha \mathrm{H} 3$ (Millipore, 05-928), $\alpha$ Daxx (Cell Signaling, 25C12), $\alpha$ Parp1 (Active Motif, 39561), $\alpha \beta$ Actin (Sigma, A5316), $\alpha \mathrm{H} 2 \mathrm{~B}$ (Millipore, 07-371), $\alpha$ Hemoglobin $\alpha$ (Santa Cruz Biotechnology, sc-21005), and $\alpha \mathrm{mH} 2 \mathrm{~A} 1$ (Abcam, ab37264).

\section{$L C-M S / M S$ and $q M S$}

Protein identification via LC-MS/MS was carried out essentially as described (Kaneko et al. 2010). qMS of mH2A1 was performed as described (Kapoor et al. 2010).

\section{Mononucleosome immunoprecipitation and MS quantification of $\mathrm{H} 3$ variants}

Mononucleosome immunoprecipitation and qMS of the histone peptides were carried out essentially as described (Viens et al. 2006; Kapoor et al. 2010). In order to quantify the ratio of H3.3 to $\mathrm{H} 3.1$ and H3.2, residue 31 was used (serine in place of an alanine, respectively). All methylated and acetylated forms of the peptides were considered.

\section{cDNA isolation and $q P C R$}

qPCR and mRNA analysis were carried out as described (Kapoor et al. 2010). cDNA expression was normalized to GAPDH levels. Primer sequences are provided in the Supplemental Material. implicate ATRX in nucleosomal association of mH2A1, which may be important for establishing and/or maintaining chromatin states.

\section{Materials and methods}

\section{Cell culture, plasmids, and shRNA}

HEK293 and HeLa cells were grown in DMEM, and K562 cells were suspension-cultured in RPMI (10\% FBS, $1 \%$ penicillin/streptomycin). GFPtagged H2A or mH2A isoforms were expressed in HEK293 cells (pEGFP-C1 or N1, respectively), HA-tagged H3.1 and H3.3 were expressed in HeLa cells (Wiedemann et al. 2010), and Flag-tagged H2A or mH2A1.2 were infected into HeLa cells (pQXCIP). Selection was carried out in either $800 \mu \mathrm{g} / \mathrm{mL}$ neomycin or $1 \mu \mathrm{g} / \mathrm{mL}$ puromycin. For shRNA studies, HEK293, HeLa1.2.11, and K562 cells were infected with lentiviral plasmids encoding ATRX shRNAs (Open Biosystems, RHS4533-NM_000489) or luciferase shRNA by standard procedures and grown in $1 \mu \mathrm{g} / \mathrm{mL}$ puromycin. GFP-tagged plasmids of ATRX (N, mid, C, and full-length; gift of D. Picketts) were transiently transfected into HEK293 cells.

\section{Chromatin-free immunoprecipitation}

Chromatin-free fractions were isolated essentially as described (Foltz et al. 2009). Material from large-scale chromatin-free immunoprecipitations was glycine-eluted, TCA-precipitated, and resolved by $4 \%-12 \%$ gel (NuPAGE, Invitrogen). Immunoprecipitations were carried out with $\alpha$ GFP beads (Vector Laboratories or Chromotek) for $3 \mathrm{~h}$ (at 250-375 mM salt). For immunoblots,
FRAP

Live-cell imaging and long-term FRAP experiments were carried out essentially as reported (Wiedemann et al. 2010). For visualization of the results, single-cell measurements were averaged and plotted together with the respective standard error for every time point.

\section{Native ChIP-seq}

Native mH2A1 ChIP (Abcam, ab37264) and Input DNA were prepared from K562 cells, and subsequent sequencing was performed using Illumina Hi-Seq. See the Supplemental Material for full details.

\section{Telomere Southern blot}

DNA was isolated following mH2A1 ChIP, and telomere Southern blot was carried out as described (Goldberg et al. 2010), with the exception that the probe used was $\mathrm{T}_{2} \mathrm{AG}_{3}$ from pSty-11.

\section{Acknowledgments}

We thank Doug Higgs, Marco De Gobbi, and the Bernstein laboratory for discussions and assistance; Aurelian Radu, Peter Warburton, Dung-Fang Lee, Eros Lazzerini Denchi, Titia de Lange, Yvette Yien, Jim Bieker, David Picketts, Dan Foltz, and Stuart Aaronson's laboratory for advice and reagents; and the Mount Sinai Institute for Genomics, Amin Mazloom, Heinrich Leonhardt, and the BioImaging Network Munich for support. This work is supported by NCI T32-CA078207 to L.F.D.; NIH MSTP T32GM007280 to D.Y.Z.; Major State Basic Research Development Program, 
China (2011CB965300), to H.L.; National Natural Science Foundation of China $(60905013,60934004$, and 91019016) to X.W.; DFG SFB TR5 to S.B.H. and SCHE1596/2-1 to L.S.; CIPSM to S.B.H.; an NIH Innovator award (DP2OD007447) and NSF Faculty Early CAREER award to B.A.G.; and The Ellison Medical Foundation New Scholar Award and NCI/NIH R01CA154683 to E.B.

\section{References}

Angelov D, Molla A, Perche PY, Hans F, Cote J, Khochbin S, Bouvet P, Dimitrov S. 2003. The histone variant macroH2A interferes with transcription factor binding and SWI/SNF nucleosome remodeling. Mol Cell 11: 1033-1041.

Banaszynski LA, Allis CD, Lewis PW. 2010. Histone variants in metazoan development. Dev Cell 19: 662-674.

Berube NG. 2011. ATRX in chromatin assembly and genome architecture during development and disease. Biochem Cell Biol 89: 435-444.

Berube NG, Smeenk CA, Picketts DJ. 2000. Cell cycle-dependent phosphorylation of the ATRX protein correlates with changes in nuclear matrix and chromatin association. Hum Mol Genet 9: 539-547.

Buschbeck M, Uribesalgo I, Wibowo I, Rue P, Martin D, Gutierrez A, Morey L, Guigo R, Lopez-Schier H, Di Croce L. 2009. The histone variant macroH2A is an epigenetic regulator of key developmental genes. Nat Struct Mol Biol 16: 1074-1079.

Chakravarthy S, Gundimella SK, Caron C, Perche PY, Pehrson JR, Khochbin S, Luger K. 2005. Structural characterization of the histone variant macroH2A. Mol Cell Biol 25: 7616-7624.

Costanzi C, Pehrson JR. 1998. Histone macroH2A1 is concentrated in the inactive X chromosome of female mammals. Nature 393: 599-601.

Costanzi C, Pehrson JR. 2001. MACROH2A2, a new member of the MACROH2A core histone family. I Biol Chem 276: 21776-21784.

De Koning L, Corpet A, Haber JE, Almouzni G. 2007. Histone chaperones: An escort network regulating histone traffic. Nat Struct Mol Biol 14: 997-1007.

Drane P, Ouararhni K, Depaux A, Shuaib M, Hamiche A. 2010. The death-associated protein DAXX is a novel histone chaperone involved in the replication-independent deposition of H3.3. Genes Dev 24: 1253-1265.

Dunleavy EM, Roche D, Tagami H, Lacoste N, Ray-Gallet D, Nakamura Y, Daigo Y, Nakatani Y, Almouzni-Pettinotti G. 2009. HJURP is a cell-cycle-dependent maintenance and deposition factor of CENP-A at centromeres. Cell 137: 485-497.

Foltz DR, Jansen LE, Bailey AO, Yates JR 3rd, Bassett EA, Wood S, Black BE, Cleveland DW. 2009. Centromere-specific assembly of CENPa nucleosomes is mediated by HJURP. Cell 137: 472-484.

Gamble MJ, Frizzell KM, Yang C, Krishnakumar R, Kraus WL. 2010. The histone variant macroH2A1 marks repressed autosomal chromatin, but protects a subset of its target genes from silencing. Genes Dev 24: 21-32.

Garrick D, Samara V, McDowell TL, Smith AJ, Dobbie L, Higgs DR, Gibbons RJ. 2004. A conserved truncated isoform of the ATR-X syndrome protein lacking the SWI/SNF-homology domain. Gene 326: $23-34$.

Gaume X, Monier K, Argoul F, Mongelard F, Bouvet P. 2011. In vivo study of the histone chaperone activity of nucleolin by FRAP. Biochem Res Int 2011: 187624. doi: 10.1155/2011/187624.

Goldberg AD, Banaszynski LA, Noh KM, Lewis PW, Elsaesser SJ, Stadler S, Dewell S, Law M, Guo X, Li X, et al. 2010. Distinct factors control histone variant H3.3 localization at specific genomic regions. Cell 140: $678-691$.

Heaphy CM, de Wilde RF, Jiao Y, Klein AP, Edil BH, Shi C, Bettegowda C, Rodriguez FJ, Eberhart CG, Hebbar S, et al. 2011. Altered telomeres in tumors with ATRX and DAXX mutations. Science 333: 425.

Higgs DR, Garrick D, Anguita E, De Gobbi M, Hughes J, Muers M, Vernimmen D, Lower K, Law M, Argentaro A, et al. 2005. Understanding $\alpha$-globin gene regulation: Aiming to improve the management of thalassemia. Ann N Y Acad Sci 1054: 92-102.

Iwase S, Xiang B, Ghosh S, Ren T, Lewis PW, Cochrane JC, Allis CD, Picketts DJ, Patel DJ, Li H, et al. 2011. ATRX ADD domain links an atypical histone methylation recognition mechanism to human mental-retardation syndrome. Nat Struct Mol Biol 18: 769-776.

Kaneko S, Li G, Son J, Xu CF, Margueron R, Neubert TA, Reinberg D. 2010. Phosphorylation of the PRC2 component Ezh2 is cell cycle- regulated and up-regulates its binding to ncRNA. Genes Dev 24: 2615-2620.

Kapoor A, Goldberg MS, Cumberland LK, Ratnakumar K, Segura MF, Emanuel PO, Menendez S, Vardabasso C, Leroy G, Vidal CI, et al. 2010. The histone variant macroH2A suppresses melanoma progression through regulation of CDK8. Nature 468: 1105-1109.

Law MJ, Lower KM, Voon HP, Hughes JR, Garrick D, Viprakasit V, Mitson M, De Gobbi M, Marra M, Morris A, et al. 2010. ATR-X syndrome protein targets tandem repeats and influences allele-specific expression in a size-dependent manner. Cell 143: 367-378.

Lewis PW, Elsaesser SJ, Noh KM, Stadler SC, Allis CD. 2010. Daxx is an H3.3-specific histone chaperone and cooperates with ATRX in replication-independent chromatin assembly at telomeres. Proc Nat1 Acad Sci 107: 14075-14080.

Mendez J, Stillman B. 2000. Chromatin association of human origin recognition complex, cdc 6 , and minichromosome maintenance proteins during the cell cycle: Assembly of prereplication complexes in late mitosis. Mol Cell Biol 20: 8602-8612.

Mitson M, Kelley LA, Sternberg MJ, Higgs DR, Gibbons RJ. 2011. Functional significance of mutations in the Snf2 domain of ATRX. Hum Mol Genet 20: 2603-2610.

Mosammaparast N, Del Rosario BC, Pemberton LF. 2005. Modulation of histone deposition by the karyopherin kap114. Mol Cell Biol 25: 1764-1778.

Novikov L, Park JW, Chen H, Klerman H, Jalloh AS, Gamble MJ. 2011. Qki-mediated alternative splicing of the histone variant Macroh2a1 regulates cancer cell proliferation. Mol Cell Biol 31: 4244-4255.

Papamichos-Chronakis M, Watanabe S, Rando OJ, Peterson CL. 2011. Global regulation of H2A.Z localization by the INO80 chromatinremodeling enzyme is essential for genome integrity. Cell 144: 200213.

Park YJ, Luger K. 2008. Histone chaperones in nucleosome eviction and histone exchange. Curr Opin Struct Biol 18: 282-289.

Pehrson JR, Fried VA. 1992. MacroH2A, a core histone containing a large nonhistone region. Science 257: 1398-1400.

Plazas-Mayorca MD, Zee BM, Young NL, Fingerman IM, LeRoy G, Briggs $\mathrm{SD}$, Garcia BA. 2009. One-pot shotgun quantitative mass spectrometry characterization of histones. J Proteome Res 8: 5367-5374.

Sporn JC, Kustatscher G, Hothorn T, Collado M, Serrano M, Muley T, Schnabel P, Ladurner AG. 2009. Histone macroH2A isoforms predict the risk of lung cancer recurrence. Oncogene 28: 3423-3428.

Tang J, Wu S, Liu H, Stratt R, Barak OG, Shiekhattar R, Picketts DJ, Yang X. 2004. A novel transcription regulatory complex containing death domain-associated protein and the ATR-X syndrome protein. I Biol Chem 279: 20369-20377.

Timinszky G, Till S, Hassa PO, Hothorn M, Kustatscher G, Nijmeijer B, Colombelli J, Altmeyer M, Stelzer EH, Scheffzek K, et al. 2009. A macrodomain-containing histone rearranges chromatin upon sensing PARP1 activation. Nat Struct Mol Biol 16: 923-929.

Viens A, Mechold U, Brouillard F, Gilbert C, Leclerc P, Ogryzko V. 2006. Analysis of human histone $\mathrm{H} 2 \mathrm{AZ}$ deposition in vivo argues against its direct role in epigenetic templating mechanisms. Mol Cell Biol 26: 5325-5335.

Wiedemann SM, Mildner SN, Bonisch C, Israel L, Maiser A, Matheisl S, Straub T, Merkl R, Leonhardt H, Kremmer E, et al. 2010. Identification and characterization of two novel primate-specific histone $\mathrm{H} 3$ variants, H3.X and H3.Y. J Cell Biol 190: 777-791.

Wilson BG, Roberts CW. 2011. SWI/SNF nucleosome remodellers and cancer. Nat Rev Cancer 11: 481-492.

Wong LH, McGhie JD, Sim M, Anderson MA, Ahn S, Hannan RD, George AJ, Morgan KA, Mann JR, Choo KH. 2010. ATRX interacts with H3.3 in maintaining telomere structural integrity in pluripotent embryonic stem cells. Genome Res 20: 351-360.

Zhang R, Poustovoitov MV, Ye X, Santos HA, Chen W, Daganzo SM, Erzberger JP, Serebriiskii IG, Canutescu AA, Dunbrack RL, et al. 2005. Formation of MacroH2A-containing senescence-associated heterochromatin foci and senescence driven by ASF1a and HIRA. Dev Cell 8: 19-30. 


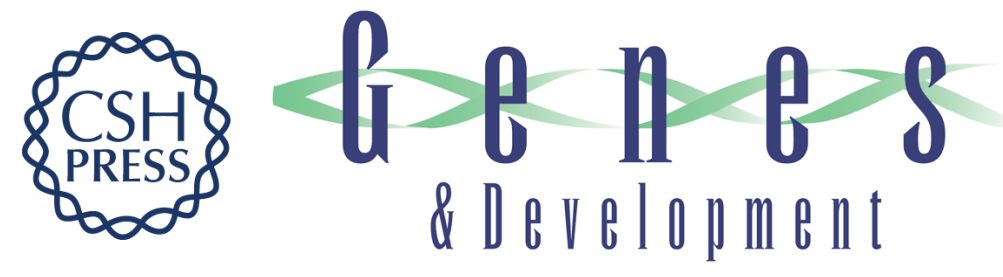

\section{ATRX-mediated chromatin association of histone variant macroH2A1 regulates $\alpha$-globin expression}

Kajan Ratnakumar, Luis F. Duarte, Gary LeRoy, et al.

Genes Dev. 2012, 26:

Access the most recent version at doi:10.1101/gad.179416.111

Supplemental
Material $\quad$ http://genesdev.cshlp.org/content/suppl/2012/03/05/26.5.433.DC1

References This article cites 39 articles, 15 of which can be accessed free at: http://genesdev.cshlp.org/content/26/5/433.full.html\#ref-list-1

License

Email Alerting Receive free email alerts when new articles cite this article - sign up in the box at the top Service 